\title{
WHO Strategy for Collecting Safety Data in Public Health Programmes: Complementing Spontaneous Reporting Systems
}

\author{
Shanthi N. Pal · Chris Duncombe • \\ Dennis Falzon $\cdot$ Sten Olsson
}

Published online: 18 January 2013

(c) The Author(s) 2013. This article is published with open access at Springerlink.com

\begin{abstract}
Globally, national pharmacovigilance systems rely on spontaneous reporting in which suspected adverse drug reactions (ADRs) are reported to a national coordinating centre by health professionals, manufacturers or patients. Spontaneous reporting systems are the easiest to establish and the cheapest to run but suffer from poorquality reports and underreporting. It is difficult to estimate rates and frequencies of ADRs through spontaneous reporting. Public health programmes need to quantify and characterize risks to individuals and communities from their medicines, to minimize harm and improve use, to sustain public confidence in the programmes, and to track problems due to medication errors and poor quality medicines. Additional methods are therefore needed to monitor the quantitative aspects of medicine safety, to better identify specific risk factors and high-risk groups, and to characterize ADRs associated with specific medicines and in specific populations. The present paper introduces two methods, cohort event monitoring and targeted spontaneous reporting, that are being implemented by the WHO, in its public health programmes, to complement spontaneous
\end{abstract}

S. N. Pal ( ()

Quality Assurance and Safety: Medicines, Essential Medicines and Health Products, WHO, 20 Avenue Appia 1211, Geneva 27, Switzerland

e-mail: pals@who.int

C. Duncombe

Bill and Melinda Gates Foundation, Seattle, WA, USA

D. Falzon

Stop TB, WHO, Geneva, Switzerland

S. Olsson

Uppsala Monitoring Centre, Uppsala, Sweden reporting. The advantages and disadvantages of these methods and how each can be applied in clinical practice are discussed.

\section{Introduction}

Pharmacovigilance (PV) is defined as the science and activities relating to the detection, assessment, understanding and prevention of adverse effects and all other problems related to medicines [1]. The 16th World Health Assembly (1963) adopted a resolution (WHA 16.36) that reaffirmed the need for early detection and rapid dissemination of information on adverse reactions due to medicines, and subsequently led to the creation of the WHO Programme for International Drug Monitoring. Under this programme, systems have been created in Member States for the collection and evaluation of individual case safety reports (ICSRs) [2].

\section{Spontaneous Reporting System}

In all countries (low, middle or high income), national PV systems rely heavily on spontaneous (or voluntary) reporting in which suspected adverse drug reactions (ADRs) are reported to a national coordinating centre by health professionals, manufacturers or directly by patients. Of all the sources of data for drug safety monitoring, the spontaneous reporting systems provide the highest volume of information at the lowest maintenance cost [3], and have proven their value in the early detection of patient safety issues related either to the products themselves or to their use [4]. The most important function of spontaneous reporting systems is the early identification of signals [5] 
and formulation of hypotheses, leading to further confirmatory investigations or sometimes regulatory warnings and changes of product information leaflets. In some instances, withdrawals of marketing authorizations are also based on ICSRs [6]. For example, in the case of cerivastatin, an association (a 'signal') between cerivastatin, myopathy and rhabdomyolysis was published, based on ICSRs, by the Uppsala Monitoring Centre (UMC) in 1999; various regulatory decisions were announced between 1999 and 2001 in different countries [7].

In spontaneous reporting, the most common form of PV, no measures are taken to systematically follow-up patients to record ADRs that they may have experienced. Reporting is dependent on the initiative and motivation of the reporters. This leads to underreporting [8] compared with actual incidence of ADRs. The absence of an effective PV system with clear protocols, tools and a PV mandate can also act as a barrier to ADR reporting [9]. Under these circumstances it is difficult to determine the actual number of individuals experiencing an adverse reaction to the medicine. This, together with the uncertainties around the number of patients exposed to the medicine in question, makes it difficult to estimate rates and frequencies of ADRs through spontaneous reporting. Methods of greater scientific rigour are needed to establish quantitative aspects of medicine safety, to better identify specific risk factors and high-risk groups, and to provide valid clinical characteristics of problems associated with specific medicines.

Public health programmes may treat a large population, in an organized and structured fashion, and record the number of patients treated, drugs used, doses given, etc. These programmes thus provide good platforms for capturing the quantitative aspects (rates and frequencies) of ADRs and adverse events with the medicines used [10]. In the management of HIV and tuberculosis (TB) patients, short- and long-term toxicity data play a critical role in informing treatment choices [11-13]. In adopting a public health approach to the delivery of HIV and TB services, it is important to quantify and characterize treatment-related risks, as early as possible, to minimize the harm to public health as well as to sustain public confidence in the programme [14]. For example, it is important to know what ADRs may be expected with certain treatment and how many will experience this ADR. Such quantitative information will have a direct impact on treatment guidelines, policies and practices. While spontaneous reporting remains the bedrock of safety monitoring, additional methods are needed to quantify the frequency and severity of expected and unanticipated ADRs occurring in patients receiving long-term treatment. In the context of HIV programmes which provide life-long therapy, a simple, inexpensive but robust methodology is needed to capture both known and unknown ADRs. In addition, we need to monitor adverse events that, instead of being directly attributed to the 'active' substance, could be attributed more to aspects such as inappropriate use, medication errors, poor quality products or drug-drug interactions. The Global Fund urges countries to invest in PV programmes that monitor the quality, usage and efficacy of the HIV, TB and malaria medicines that it helps countries to buy [15]. An effective PV programme is one that monitors medicines used in all public health programmes, to improve patient safety, and to improve the credibility and public perception of these programmes.

\section{Methods that Complement Spontaneous Reporting Systems}

\subsection{Cohort Event Monitoring (CEM)}

Cohort Event Monitoring (CEM) [16, 17] is a prospective, observational, cohort study of adverse events associated with one or more medicines. An adverse event is any untoward medical occurrence that may present during treatment with a pharmaceutical product, but which does not necessarily have a causal relationship with this treatment. A CEM programme is essentially an observation of a new medicine in routine clinical practice in the early postmarketing phase, but it can be used for older medicines. It is based on the principles of the New Zealand Intensive Medicines Monitoring Programme [18] and the UK Prescription Event Monitoring [19] except that in most resource-limited countries, treatment within public health programmes (such as TB and HIV) is not provided on a prescription basis. CEM is thus an early warning system that interviews patients on a certain treatment (the cohort), for capturing problems (the events) with new medicines in public health programmes; the patients are interviewed before and after starting treatment. CEM captures all medicine-related events, including medication errors, problems due to poor storage conditions, poor quality or counterfeit medicines, and drug interactions. Patients may be recruited from all health facilities involved in providing the medicines, or patients may be recruited from selected health facilities that are representative of the whole country.

CEM involves recording all events, regardless of its severity or seriousness, and includes the following steps:

- A patient cohort is established for the medicine of interest.

- Adverse events experienced by patients in the cohort are recorded by interviewing patients as part of routine patient/health professional encounters.

- Patient ID and demographic data, together with details of medicines, events and other relevant information, are recorded on questionnaires. 
It is normally recommended that a cohort of 10,000 patients be enrolled $[16,17]$. This gives a $95 \%$ chance of identifying a specific event that has an incidence of $\geq 1: 3000$ (that is, an uncommon or rare event). A cohort of 3000 patients gives a $95 \%$ chance of identifying a single incident with an incidence of $\geq 1: 1000$. For a meaningful assessment, at least three events need to be identified, hence the higher objective of 10,000 patients $[16,17]$.

For most medicines, the duration of monitoring is limited. It is determined by the length of treatment to be monitored in individual patients, and the time it takes to reach the desired cohort size. In TB therapy, treatment of individual patients is monitored for a period that is considered to be appropriate for the identification of both short- and long-term effects (e.g. for drug-resistant TB this might be for 2 years or more after ending a treatment course lasting 2 years). Enrolment will continue until the target cohort population is reached. If there is special interest in subgroups such as pregnant women or children, or more information is needed on a type of event of particular concern, the programme may need to continue for a longer period to accrue sufficient numbers in these subgroups to be able to achieve statistical significance when testing associations. The data are reviewed at regular intervals (e.g. 3-monthly) to detect early trends that may require further monitoring.

Adverse events are recorded using treatment initiation and treatment review forms, to record events happening to patients during a control period, prior to and after treatment initiation, respectively. The length of the control period could vary but should not extend beyond what the patient may reasonably remember. By collecting data on events happening to patients before treatment, a baseline is created against which the events encountered during treatment may be compared.

CEM might involve special ethical considerations since it is a methodology that requires the collection of detailed personal data, for patient follow-up. Since CEM aims to measure incidence, it is essential that duplicate entries are avoided; this can be done only if patients can be identified correctly. As a prerequisite to collecting patient data, it is important to seek the approval of the highest appropriate authority in the country. Some countries may choose to obtain informed consent from every patient; however, this will be time consuming. An alternative to obtaining informed consent is the 'opt out principle' where information about the CEM programme is provided publicly, and patients can then decide whether or not they wish to have their data stored as part of CEM. These approaches have been endorsed by competent authorities internationally [20]. Systems for data storage should be password protected, with controlled access to the premises, etc. The UMC has, in collaboration with the WHO, developed a specific software, CemFlow ${ }^{\mathrm{TM}}$, for the secure management and analysis of CEM data. It is web-based but data entry can be done offline with regular uploads to the main database.

Detailed handbooks describing these principles for CEM in public health programmes have been developed by the WHO [16, 17] and can be downloaded from http://www. who.int/medicines/areas/quality_safety/safety_efficacy/ pharmpubhealth/en/index.html.

CEM is currently being used for antimalarials in Kenya, Nigeria, the United Republic of Tanzania, and Zimbabwe. CEM of antiretroviral (ARV) drugs is planned in Belarus and in the United Republic of Tanzania. CemFlow ${ }^{\mathrm{TM}}$ is the support system used for the implementation of CEM in these countries.

\subsection{Targeted Spontaneous Reporting (TSR)}

The WHO proposes targeted spontaneous reporting (TSR) as a methodology that builds on the principles of spontaneous reporting but applied in a defined setting [21]. In this method, health professionals managing a well-defined group of patients [e.g. patients on treatment for drugresistant TB or those switching from first-line to secondline antiretroviral therapy (ART)] are sensitized to report specific safety concerns suspected to be medicine related. TSR addresses a distinct set of questions and provides a comprehensive monitoring method that is affordable, feasible and sustainable in settings with limited financial and human resources. It also promotes the role of PV as a best practice that improves quality of care.

TSR was developed by the WHO in 2010 and is being piloted in the HIV treatment programmes in three countries (Kenya, Vietnam and Uganda). TSR may be adapted either to report all suspected reactions in the defined population or to focus only on specific reactions of particular concern, for example treatment-threatening toxicity, etc. This serves to limit the reporting workload to those adverse events that are most significant to individuals and to the programmes. Poor adherence to treatment due to adverse events such as nausea can be included as one of the targeted events in the TSR concept for priority reporting. It is particularly useful in the targeted follow-up of patients with additional complications, such as patients with drug-resistant $\mathrm{TB}$ and lifelong ART. In HIV care, compared with routine spontaneous reporting, TSR aims to increase reporting rates by targeting, training and mentoring reporters at selected high caseload clinics, and by task shifting the reporting to nonphysician cadres of healthcare workers such as nurses, pharmacists, pharmacy technicians and patients living with HIV (PLHIV). By monitoring populations such as those receiving extended ARV prophylaxis to prevent breast milk transmission of HIV, and by focusing on particular 
ADRs of interest, such as renal toxicity related to tenofovir, reporting is kept simple and feasible without compromising quality.

The WHO is now aiming to introduce TSR in TB treatment programmes. For many years, national TB programmes monitor patient outcomes in well-circumscribed groups termed cohorts. TSR can integrate monitoring of ADRs as a standard of care, alongside the routine practice of monitoring success, death, default or failure of treatment within these TB cohorts. One benefit of monitoring within a treatment cohort is that the number and profiles of the exposed patients will be known. The number of patients in the treatment cohorts represent a denominator; if all suspected cases of the targeted ADR within this cohort are recorded, then it is possible to calculate simple frequencies of the targeted ADR.

TSR thus seeks to overcome the main disadvantages of spontaneous reporting, that is underreporting and the inability to calculate rates, while maintaining simplicity of use, low cost and linkage to existing systems. In all public health programmes reporting of drug-related adverse events is extremely low. More than 100 countries are members of the WHO PV programme [2] and have existing systems in place for reporting ADRs but do not always integrate with public health programmes [9]. One of the objectives of TSR is to address this lack of integration between PHPs and PV programmes by leveraging existing PV systems, providing simple tools to PHPs, training and task shifting to encourage reporting.

The steps required to meet the objectives of TSR include the following:

- Monitoring for suspected drug-related problems is included as part of normal patient care. During patient investigation the possibility of a medicine-related problem is always considered. At every encounter, the healthcare professional screens for any suspected ADR.

- Suspicion of the possibility of a causal relationship between drug treatment and the event triggers the completion of an ADR reporting form. The suspected ADR is noted in the patient records.

- All healthcare professionals involved in patient care are sensitized to ask about and investigate adverse effects at every encounter.

- The reporting forms (the generic national ADR form) and route for transmission of information are the same as those used in spontaneous reporting, but the forms are supported by specific guidance (case definitions and written procedures) on when to complete them and with details on standardized reporting of drug names and ADRs.

- Reporting may primarily target serious or treatment threatening/limiting (treatment discontinuation, change of regimen, life-threatening event, hospitalization, poor adherence because of toxicity or death) ADRs rather than the notification of any suspected reaction. TSR can be adapted to the safety question at hand. If the total burden of drug-related problems in the exposed population is of interest, health professionals can be instructed to report any suspected drug-related problem. If, however, the frequency of a specific problem suspected to be associated with the therapy given is the important question, e.g. vision disorders, a case definition for reporting can be given in the instructions to healthcare professionals.

- Reporting lasts the whole length of treatment. In the case of TB, this is between 6 months and 2 years, and in the case of ART, lifelong

- Unlike CEM, there are no baseline measurements nor is there any active follow-up of members of the cohort and, thus, less resources are required.

In public health programmes that integrate TSR, the routine patient record will include the question "Suspected adverse drug reaction: Yes or No?", thereby ensuring that it is always considered. The extent to which this information is recorded will indicate whether ADR monitoring has become a part of normal practice. If safety monitoring of each patient is truly part of best practice and recording of whether the patient has experienced a suspected medicinerelated problem or not is complete, the calculated reaction frequencies may be close estimates of true incidence rates.

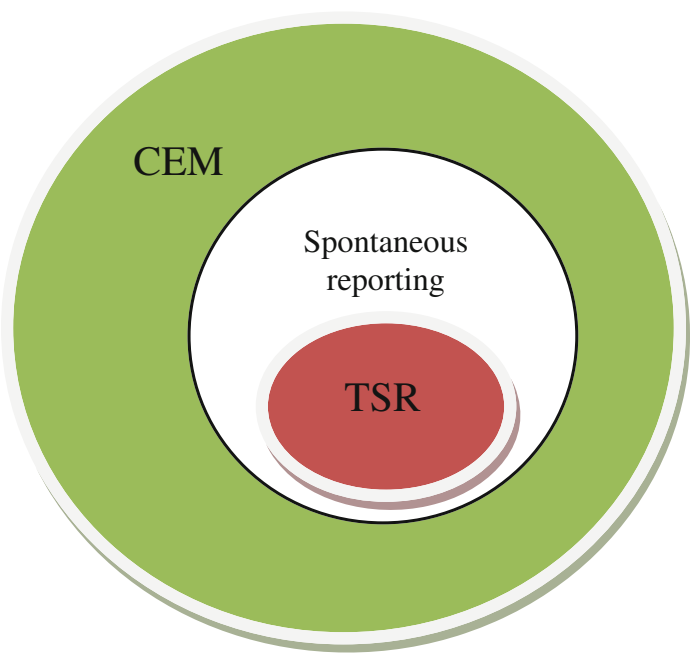

Fig. 1 The relationship between spontaneous reporting, TSR and CEM. All medical incidents (events) that patients experienced while on treatment can be captured by CEM. Those events considered noxious and unintended and suspected to be caused by the medicine are reportable as ADRs through spontaneous reporting. TSR focuses on the collection of information on specific ADRs, with specific medicines, in defined patient groups. ADRs adverse drug reactions, CEM cohort event monitoring, TSR targeted spontaneous reporting 
Table 1 Advantages and disadvantages of different types of PV methods

\section{Spontaneous reporting}

Advantages

Administratively simpler and less labour-intensive than CEM

Less costly than CEM

Has the potential of identifying very rare problems related to medicine use in any healthcare setting

PV centres and health professionals are more likely to be familiar with this method as it is the most common method of PV used

Provides safety surveillance throughout the marketed life of all medicines

Disadvantages

The data collected by this method are incomplete both in terms of quality and quantity

Underreporting is significant and widespread

Reliable rates cannot be calculated and so risk cannot be measured and risk factors cannot be established with confidence

There are strong biases in reporting

Deaths due to ADRs may be incompletely recorded/investigated in some countries, especially when they occur outside healthcare

Special studies will need to be set up to obtain accurate information on areas of particular interest, e.g. pregnancy, children and specific events of concern. These special studies add to the cost and in turn reduce the cost advantage of spontaneous reporting

\section{CEM}

Advantages

The ability to produce rates

The ability to produce a near-complete profile of the adverse events and/or ADRs for the medicines of interest

Very effective in identifying signals at an early stage

The ability to associate reactions with risk factors

The ability to make accurate comparisons between medicines

Can detect reduced or failed therapeutic effect and can raise suspicion of medication errors, interactions, emerging resistance or poor-quality or counterfeit medicines

The ability to record and examine details of all deaths and provide rates of death

Disadvantages

The method is more labour-intensive, needs dedicated staff to perform treatment initiation (baseline) and treatment follow-up interviews

More costly than spontaneous reporting

Patients may not turn up for follow-up; potential for loss to follow-up

Patients may 'opt-out' and refuse to be part of the CEM; this might make it difficult to reach the required cohort size

Takes certain expertise in recording adverse events, training will be necessary

Cannot detect very rare problems with medicines

TSR

Advantages

Is simpler, less costly and less labour-intensive than CEM

TSR represents an 'add-on' to the routine monitoring of outcomes of patients

Can be focused on priority ADRs

The forms and routes for reporting are similar to those for routine spontaneous reporting

Can provide some measure of rates and incidences

Uses existing PV systems within countries

Links public health programmes to PV centres

Less likely to identify unanticipated reactions

Disadvantages

The method is subject to individual willingness to monitor and report; thus numerator (number of individuals with the suspected ADR) may not be accurate

Completeness of reporting is therefore crucial

There is limited experience with TSR and the technique needs to be field-tested

ADRs adverse drug reactions, CEM cohort event monitoring, $P V$ Pharmacovigilance, TSR targeted spontaneous reporting 
TSR provides the opportunity to monitor every single patient on treatment, as part of treatment and care. However, its successful implementation depends on the willingness of healthcare providers to participate in this monitoring exercise and to report their observations. ADR reporting can be improved through interventions such as education, advocacy, mentoring, etc [22, 23]. Although different from these approaches, TSR, with its focus on targeted ADRs and/or medicines, is likely to have a similar (positive) impact on reporting, albeit only for the targeted ADRs, by reducing the reporting burden on overworked health professionals.

Figure 1 highlights the relationship between spontaneous reporting, CEM and TSR. Table 1 summarizes the comparative advantages and disadvantages of the three methods.

\section{CEM and TSR Projects in Other Countries}

The WHO has organized training courses in TSR (Botswana, Kenya, Uganda and Zimbabwe) and CEM (Belarus, Burkina Faso, Ethiopia, Ghana, Kenya, Nigeria, Republic of Moldova, Ukraine, Uganda, United Republic of Tanzania, and Zambia). As mentioned above, several of these countries are in the process of implementing projects, either in CEM or TSR, with technical support from the WHO, and with funds from the European Commission, the Bill and Melinda Gates Foundation and others.

Preliminary feedback and data from these efforts are encouraging and there is general enthusiasm and interest in adopting these methods in other countries.

\section{Conclusion}

PV is an essential component of patient care and surveillance. It aims to achieve the best outcome from treatment with medicines and medicinal products. Good PV will identify the risks within the shortest possible time after the medicine has been marketed and will help establish or identify risk factors. When communicated effectively, this information allows for rational, evidence-based prescribing with the potential for preventing many ADRs. Such information will ultimately help each patient receive optimum therapy at a lower cost to the health system. The organizers of a monitoring programme for the safety of medicines used in public health programmes must have a clear sense of the questions they want to answer before developing their plan. Routine safety monitoring, as a lifecycle approach for the product, is best handled by a spontaneous reporting system. But if the aim is to better understand, with minimum resources, the occurrence of a specific ADR in a specific population, TSR is an appropriate choice. If the aim is to actively follow patients to characterize the safety profile of new medicines, then CEM is a relevant choice. It is only with clear goals in mind that one can design a suitable data collection instrument and an analytical plan. Whatever the method, all stakeholders must be fully informed of the reasons for monitoring, the methodology as far as it involves them, the value of safety monitoring, the contribution it will make to the health of the population, the potential for increasing the effectiveness of public health programmes and reducing health costs, and the contribution PV can make to the knowledge of medicines in public health programmes and their safety at country and global levels.

Acknowledgements No external sources of funding were used to assist in the preparation of this manuscript. The authors have no conflicts of interest that are relevant to the contents of this manuscript. Dr. Chris Duncombe, currently employed with the Gates Foundation, contributed to this article in his previous capacity, as a WHO staff member in the HIV AIDS department, from 2010-2011.

Open Access This article is distributed under the terms of the Creative Commons Attribution Noncommercial License which permits any noncommercial use, distribution, and reproduction in any medium, provided the original author(s) and the source are credited.

\section{References}

1. WHO. The importance of pharmacovigilance: safety monitoring of medicinal products. Geneva: WHO; 2002.

2. Pal S, Dodoo A, Mantel A, et al. Pharmacovigilance and safety of medicines. In: WHO, editor. The world medicines situation 2011. Geneva: WHO; 2011.

3. Sharma M, Gupta SK. Postmarketing surveillance. In: Gupta SK, editor. Textbook of pharmacovigilance. New Delhi: Jaypee Brothers; 2011. p. 75-92.

4. Alj L, Touzani MDW, Benkirane R, et al. Detecting medication errors in pharmacovigilance database: capacities and limits. Int J Risk Saf Med. 2007;19(4):187-94.

5. Härmark L, van Grootheest AC. Pharmacovigilance: methods, recent developments and future perspectives. Eur J Clin Pharmacol. 2008;64(8):743-52.

6. Clarke A, Deeks JJ, Shakir SA. An assessment of the publicly disseminated evidence of safety used in decisions to withdraw medicinal products from the UK and US markets. Drug Saf. 2006;29(2):175-81.

7. WHO. WHO international drug monitoring: cerivastatin and gemfibrozil. WHO Drug Info. 2002;16(2):8-11.

8. Hazell L, Shakir SA. Under-reporting of adverse drug reactions: a systematic review. Drug Saf. 2006;29:385-96.

9. Olsson S, Pal S, Stergachis A, et al. Pharmacovigilance activities in 55 low- and middle-income countries: a questionnaire-based analysis. Drug Saf. 2010;33(8):689-703.

10. WHO. The safety of medicines in public health programmes: pharmacovigilance an essential tool. Geneva: WHO; 2006.

11. WHO. Antiretroviral drugs for treating pregnant women and preventing HIV infection in infants: recommendations for a public health approach (2010 version). Geneva: WHO; 2010. 
12. WHO. Treatment of tuberculosis: guidelines, 4th edn. Geneva: WHO; 2010.

13. Falzon D, Jaramillo E, Schünemann HJ, et al. WHO guidelines for the programmatic management of drug-resistant tuberculosis: 2011 update. Eur Respir J. 2011;38:516-28.

14. Dodoo A, Adjei S, Couper M, et al. When rumours derail a mass deworming exercise. Lancet. 2007;370:465-6.

15. Global Fund to Fight AIDS, TB and Malaria. The Global Fund strategy 2012-2016: investing for impact. Geneva: Global Fund to Fight AIDS, TB and Malaria; 2012.

16. WHO. A practical handbook on the pharmacovigilance of antimalarial medicines. Geneva: WHO; 2008.

17. WHO. A practical handbook on the pharmacovigilance of antiretroviral medicines. Geneva: WHO; 2009.

18. Coulter DM. The development of prescription event monitoring in the New Zealand Intensive Medicines Monitoring Programme.
In: Mann R, Andrews EB, editors. Pharmacovigilance. Chichester: Wiley; 2002. p. 345-62.

19. Shakir SAW. Prescription-event monitoring. In: Strom BL, editor. Pharmacoepidemiology. 4th ed. Chichester: Wiley; 2005. p. $337-46$.

20. Council for International Organizations of Medical Sciences (CIOMS). International ethical guidelines for epidemiological studies. Geneva: CIOMS; 2009.

21. WHO. A practical handbook on the pharmacovigilance of medicines used in the treatment of tuberculosis. Geneva: WHO; 2012.

22. Clarkson A, Ingleby E, Choonara I, et al. A novel scheme for the reporting of adverse drug reactions. Arch Dis Child. 2001;84(4):337-9.

23. Mehta U, Durheim D, Mabuza A, et al. Malaria pharmacovigilance in Africa: lessons from a pilot project in Mpumalanga province, South Africa. Drug Saf. 2007;30(10):899-910. 